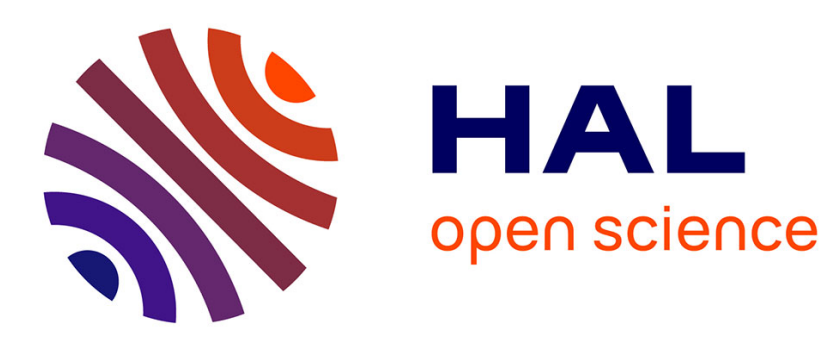

\title{
Allotissement et rendements d'échelle: application aux réseaux de transport ferroviaire
}

\author{
Julien Lévêque
}

\section{To cite this version:}

Julien Lévêque. Allotissement et rendements d'échelle: application aux réseaux de transport ferroviaire. Economie et Prévision, 2007, 178-179, pp. 147-159. halshs-00280396

\section{HAL Id: halshs-00280396 \\ https://shs.hal.science/halshs-00280396}

Submitted on 27 May 2009

HAL is a multi-disciplinary open access archive for the deposit and dissemination of scientific research documents, whether they are published or not. The documents may come from teaching and research institutions in France or abroad, or from public or private research centers.
L'archive ouverte pluridisciplinaire HAL, est destinée au dépôt et à la diffusion de documents scientifiques de niveau recherche, publiés ou non, émanant des établissements d'enseignement et de recherche français ou étrangers, des laboratoires publics ou privés. 


\title{
Allotissement et rendements d'échelle
}

\section{Application aux réseaux de transport ferroviaire}

\author{
Julien Lévêque ${ }^{(*)}$
}

Le XX $X^{e}$ siècle a vu les systèmes ferroviaires européens converger vers un modèle d'organisation monopolistique, public, intégré. Malgré les changements survenus dans la demande de transport et l'analyse économique, la sclérose de ces systèmes nationaux a conduit à de multiples crises financières durant les années quatre-vingt-dix.

Dans ce contexte, la nouvelle réglementation européenne conduit à repenser les systèmes ferroviaires en les ouvrant davantage à la concurrence, sur la base d'une séparation entre les activités de transport et de gestion de l'infrastructure. Ainsi les services de Transports express régionaux (TER), déjà régulés par les régions, ont vocation à être délégués par voie d'appel d'offre à d'autres opérateurs, au-delà de l'actuelle SNCF. Dans cette optique, le travail de recherche présenté dans cet article vise à offrir aux régions une aide à la décision en ce qui concerne le périmètre optimal des réseaux de services TER à déléguer : quel monopole naturely a-t'il dans les services de transports régionaux? Doit-on allotir les réseaux de services TER lors des futurs appels d'offre? Comment définir les lots à ouvrir à la concurrence?

Pour répondre à ces questions, la démarche adoptée mobilise deux axes de la littérature économique.

Tout d'abord, l'article s'inscrit dans la démarche normative issue de la nouvelle économie de la réglementation. L'analyse est notamment développée sur la base des éléments mis en évidence par Gasmi, Laffont et Sharkey dans leur article «The natural monopoly test reconsidered 》 de 2002. Selon ces auteurs, le périmètre du monopole naturel doit être défini en intégrant-outre la croissance des rendements d'échelle - le haut niveau de rentes informationnelles en situation monopolistique. En effet, la structure de coûts est endogène à l'organisation institutionnelle des marchés et les bénéfices de la concurrence peuvent compenser la perte des rendements d'échelle. En ce qui concerne les TER, nous reprenons l'idée selon laquelle seul un allotissement des réseaux régionaux permet de réduire les barrières à l'entrée et de favoriser la réduction des rentes informationnelles grâce à une concurrence effective.

En plus de ces deux paramètres essentiels, nous introduisons dans l'évaluation les effets de réseaux et les coûts de transaction qu'un allotissement des réseaux est susceptible de générer. Les effets de réseau sont maximisés par l'unicité des systèmes informationnels, tarifaires et commerciaux, alors que leur multiplicité engendre des coûts d'interconnexion pour les usagers. Les coûts de transaction, eux, augmentent lorsque le régulateur régional doit coordonner les différents lots afin de préserver la cohérence du réseau TER.

Par ailleurs, l'analyse des rendements d'échelle renvoie également à toute la littérature traitant ce sujet appliqué aux transports. Depuis une vingtaine d'années, les chercheurs ont notamment mis en évidence différents types de rendements d'échelle. Les rendements de taille sont estimés

(*) CNRS (Laboratoire d'Économie des Transports - Lyon) et Réseau Ferré de France (Paris).

E-mail: jleveque.research@gmail.com

Je remercie Yves Croissant et Iragaël Joly pour leurs conseils, ainsi que deux rapporteurs anonymes de cette revue. Cette recherche a été menée grâce à un financement BDI du CNRS. Les résultats et leur interprétation n'engagent que l'auteur. 
à partir de l'élasticité des coûts aux trafics, mais aussi à la longueur du réseau; ce sont eux qui permettent de répondre aux questions posées.

Enfin, l'analyse quantitative fait appel aux références classiques de l'économétrie des données de panel. Le fait que la longueur des lignes soit constante dans le temps contraint fortement le choix des estimateurs susceptibles d'être efficaces. Cela conduit à développer une méthodologie économétrique originale, justifiée par de nombreux tests statistiques.

Loin des résultats déjà connus, l'article se concentre sur certaines spécificités permettant de répondre aux objectifs fixés. En premier lieu l'analyse focalise sur l'estimation des rendements d'échelle. Il est en effet supposéque les coûts de transaction devraient rester modestes et que les coûts d'interconnexion devraient être faibles avec un allotissement pertinent. La réduction de l'inefficience engendrée par une mise en concurrence réelle est évaluée à partir de travaux précédents sur la concurrence par comparaison.

En second lieu, l'analyse des rendements d'échelle porte sur des échantillons de données fins, adaptés aux questions posées. Il ne s'agit pas d'estimer les rendements d'échelle sur la base d'une fonction de coût macro, mêlant plusieurs activités disparates (fret, TGV, transport régional, gestion de l'infrastructure) sur une étendue nationale; cela n'a plus beaucoup de sens dans l'actuel contexte de libéralisation. Au contraire le travail porte d'abord sur un échantillon de données régionales de la période 1992-1998, puis, de façon plus détaillée, sur un ensemble de sous-réseaux cohérents, constitués pour l'occasion.

Les résultats de cette estimation des rendements d'échelle sur les deux échantillons de données permettent de répondre aux questions posées. À l'échelle régionale, les rendements d'échelle estimés sont plutôt croissants, sans que ce résultat soit significatif. Compte tenu des gains élevés qu'ily aurait à faire jouer la concurrence sur des réseaux de plus petite taille, disputables par de nouveaux entrants, nous préconisons l'allotissement des réseaux TER au sein de chaque région administrative.

L'échantillon plus fin, constitué de sous-réseaux permet de cerner comment allotir de façon optimale ces réseaux régionaux. Selon la taille des régions, allotir le réseau en trois à dix sous-réseaux paraît être un compromis correct entre les rendements d'échelle (significativement croissants à ce niveau), la disputabilité de ces lots et les coûts d'interconnexion et de transaction. Cela correspond d'ailleurs approximativement à l'allotissement réalisé par les Länder allemands.

Bien sûr, ces résultats sont conditionnés par certaines limites, comme la qualité des données comptables utilisées, ou encore l'endogénéité de la fonction de coût estimée. En effet, sur la période 1992-1998 étudiée, le marché analysé est monopolisé par la SNCF et, dans ce contexte, financièrement déséquilibré à moyen terme. Si les résultats obtenus devraient être robustes aux évolutions survenues depuis, ils devraient être sensiblement modifiés par l'arrivée de nouveaux entrants. Aussi, lorsque la concurrence sera effective sur les marchés TER et que les coûts auront convergés vers ceux du marché, une nouvelle analyse permettra de réactualiser ces résultats.

Les résultats dégagés de cette transposition de la grille d'analyse établie par Gasmi, Laffont et Sharkey fournissent quelques fondements normatifs aux décisions politiques que les responsables régionaux devront bientôt prendre. Cette démarche permet enfin de mieux comprendre pourquoi et comment l'oligopole peut être collectivement préférable au monopole dans les industries de réseau. Afin de renforcer cette compréhension des systèmes économiques à rendements d'échelle supposés croissants, il conviendrait de multiplier ce type d'analyse marché par marché. Dans le cas de l'industrie ferroviaire, le même travail appliqué aux activités de gestion de l'infrastructure promet notamment d'être riche d'enseignements. 


\section{Allotir des réseaux de transport}

La nouvelle réglementation européenne conduit à déléguer certaines activités ferroviaires comme le transport régional de voyageurs, à l'issue d'une mise en concurrence pour le marché. En France, ces marchés régionaux des services TER (Transports Express Régionaux) sont encore monopolisés par l'opérateur historique. Depuis quelques années, ils sont régulés par les régions administratives ${ }^{(1)}$ qui définissent et financent les services de transport qu'elles jugent utiles. Au sein de chaque réseau régional TER, la définition des différents marchés à déléguer reste à préciser : la région doit-elle lancer un unique appel d'offre pour l'ensemble de son réseau? Doit-elle au contraire allotir le réseau et lancer un appel d'offre pour chaque lot?

Traditionnellement, l'analyse des rendements d'échelle au moyen de fonctions de coût renseigne sur le périmètre d'activité optimal d'une industrie de réseau comme les chemins de fer. Les économies d'échelle ont d'ailleurs été longtemps associées à l'idée de monopole naturel. Elles n'en sont pourtant qu'une condition suffisante (Braeutigam, 1989), dans le cas où la structure de coût est supposée exogène à la structure du marché, ce qui constitue une hypothèse très forte. Gasmi et alii (2002) montrent en effet que les rendements d'échelle «peuvent être compensés par les bénéfices d'un marché duopolistique, en termes de surplus social, de production plus importante et d'incitations plus fortes à l'efficience» ${ }^{(2)}$. Dans leur article, ces auteurs recommandent de ne pas fonder un éventuel équilibre monopolistique sur la seule analyse des rendements d'échelle.

Ainsi, en termes économiques, quatre facteurs contribuent à esquisser quelques éléments de réponse à la problématique de l'allotissement des réseaux :
- les rendements d'échelle : il s'agit d'abord de tenir compte de la structure des coûts, afin que la partition des réseaux n'engendre pas de déséconomies d'échelle par la perte du bénéfice des rendements croissants. Un tel coût réduirait alors le bien-être des contribuables qui financent l'essentiel des services TER ;

- les effets de réseau : il faut ensuite maximiser les externalités positives qui résultent de l'unicité des systèmes tarifaires, informationnels et commerciaux. La multiplicité de ces systèmes sur un même trajet induit des "coûts d'interconnexion» pour les usagers, susceptibles de les dissuader d'emprunter les TER ;

- la contestabilité des marchés : il s'agit de veiller au fait que les marchés soumis à appel d'offre puissent être véritablement disputés à l'opérateur historique par de nouveaux entrants ${ }^{(3)}$. La concurrence qui s'instaure alors incite à l'effort et réduit les rentes informationnelles du monopole; elle soulage ainsi les finances publiques régionales, au détriment du bien-être des entreprises ;

- l'organisation des marchés : il convient enfin de tenir compte des coûts de transaction occasionnés par les procédures d'appel d'offre et les suivis contractuels. Plus les marchés délégués sont nombreux, plus la gestion de l'ensemble est coûteuse, ce qui contribue à réduire le surplus social.

Le tableau 1 de variation suivant résume les enjeux, en termes de coûts et de bien-être, des décisions relatives à l'allotissement des réseaux régionaux délégués.

Il apparait ainsi qu'a priori seules les incitations concurrentielles à réduire les coûts plaident en faveur de l'allotissement des réseaux. Cependant, les coûts d'interconnexion et les coûts de transaction susceptibles d'être engendrés par l'allotissement devraient rester extrêmement modestes, par rapport au gain attendu de l'ouverture à la concurrence. Il reste donc à évaluer l'ampleur des déséconomies d'échelle potentielles pour juger de la pertinence de l'allotissement des services régionaux ${ }^{(4)}$.
Tableau 1 : variation des coûts (et surplus impactés) en fonction de la taille des réseaux délégués

\begin{tabular}{|c|c|c|}
\hline $\begin{array}{l}\text { Coûts } \\
\text { (surplus impacté) }\end{array}$ & $\begin{array}{l}\text { Monopole } \\
\text { régional }\end{array}$ & $\begin{array}{l}\text { Allotissement } \\
n \text { sous-réseaux }\end{array}$ \\
\hline & & $\begin{array}{r}\stackrel{P}{\text { Perte des rendements }} \longrightarrow \text { croissants } \\
\longrightarrow \text { con }\end{array}$ \\
\hline $\begin{array}{l}\text { Déséconomies d'échelle } \\
\text { (contribuable) }\end{array}$ & $\begin{array}{l}\text { Bénéfice des rendements } \\
\text { croissants }\end{array}$ & \\
\hline \multirow[b]{2}{*}{$\begin{array}{l}\text { Coûts d'interconnexion } \\
\text { (voyageurs) }\end{array}$} & \multirow[b]{2}{*}{$\begin{array}{l}\text { Unicité } \\
\text { des systèmes }\end{array}$} & $\begin{array}{r}\text { Multiplicité des } \\
\longrightarrow \text { systèmes }\end{array}$ \\
\hline & & \\
\hline $\begin{array}{l}\text { Rentes informationnelles } \\
\text { (SNCF) }\end{array}$ & $\begin{array}{l}\text { Inefficience, excédent } \\
\text { organisationnel }\end{array}$ & \\
\hline $\begin{array}{l}\text { Coûts de transaction } \\
\text { (contribuables) }\end{array}$ & 1 contrat & \\
\hline
\end{tabular}


Ainsi, en cas de rendements d'échelle très élevés, la configuration monopolistique de ces marchés régionaux devrait être préservée, au détriment initialement - de la concurrence. En revanche, si ces rendements s'avéraient constants ou même faibles, les gains de l'ouverture à la concurrence compenseront largement les quelques déséconomies d'échelle, grâce à un allotissement pertinent des réseaux.

Dans cet article, nous concentrons l'analyse sur les rendements d'échelle avant de discuter dans quelle mesure les résultats obtenus sont compatibles avec les effets de réseau, la contestabilité des marchés, et des coûts de transaction réduits.

La partie suivante discute l'approche traditionnelle des rendements d'échelle ferroviaires dans la littérature, avant de montrer qu'elle n'est plus appropriée lorsque l'on considère des marchés européens. La théorie relative aux concepts et à la méthodologie de l'estimation des rendements d'échelle est ensuite rappelée. La troisième partie présente l'estimation avec des modèles à erreur composée des fonctions de coût d'où seront déduits les rendements d'échelle estimés. La quatrième partie interprète économiquement les rendements d'échelle en justifiant l'allotissement des réseaux régionaux, puis en discutant une proposition d'allotissement de ces réseaux en fonction des différents facteurs précisés dans le tableau ci-dessus. Enfin, la dernière partie conclut sur la portée des résultats obtenus avec une proposition d'aide à la décision publique.

\section{L'analyse des rendements d'échelle ferroviaires}

L'analyse théorique des rendements d'échelle a été fortement développée de 1975 à 1985, période correspondant aux débuts de la libéralisation des industries de réseau aux États-Unis. Il s'agissait alors essentiellement de rendre compte des avantages structurels des compagnies de transport, liés au volume de leur production et à l'étendue de leur réseau.

Depuis les travaux fondateurs de Caves, Christensen et Tretheway (Caves et alii, 1984 ; Caves et alii, 1985), l'approche adoptée dans la littérature repose sur une analyse macroéconomique, agrégée, à l'échelle des différents réseaux de transport. À la suite des travaux américains conduits par ces auteurs, les rendements d'échelle des compagnies européennes intégrées de chemins de fer ont été analysés, notamment par Cantos (2001). Enfin, Ida et Suda (2004) ont récemment étudié les structures de coût des six principales compagnies japonaises.
Ces travaux concluent généralement à l'existence d'une certaine forme de rendements d'échelle : les rendements de densité, c'est-à-dire que pour un réseau de services identiques, une hausse du trafic engendre une augmentation proportionnellement moindre des coûts totaux. En revanche, la plupart des études ne permettent pas de prouver qu'il y a un gain, sous forme d'économies d'envergure, à multiplier les différents types de trafic, qu'il s'agisse de voyageurs et de marchandises en Europe, ou de voyageurs à grande vitesse et à vitesse classique au Japon.

Cette approche agrégée traditionnelle n'est cependant plus très pertinente en Europe, compte tenu du contexte de libéralisation des marchés ferroviaires. En effet, les réformes engagées ont conduit à rendre les entreprises ferroviaires européennes significativement hétérogènes. Il en résulte que leurs fonctions de coût ne peuvent plus être estimées à partir d'une même spécification agrégée, ceci pour les deux principales raisons suivantes :

- d'une part, l'agrégation verticale des données, correspondant à l'intégration des différentes activités ferroviaires (transport de marchandises, transport de voyageurs, gestion de l'infrastructure), n'est plus pertinente. En effet, avec la disparition des entreprises ferroviaires intégrées et l'ouverture des marchés, les périmètres de l'exercice de ces activités ne se superposent plus : certains opérateurs historiques ont vendu leur activité de transport de marchandises, d'autres ont dû céder leur activité de gestionnaire d'infrastructure. Rappelons également que cette agrégation verticale, lorsqu'elle est envisageable, soulève le problème de la pondération entre les produits (quelle équivalence en termes de coût entre un voyageur.kilomètre et une tonne.kilomètre?);

- d'autre part, l'agrégation horizontale des données, correspondant à l'intégration des trafics à l'échelle nationale (transports régionaux, longue distance, grande vitesse), conduit à estimer des fonctions de coût sur la base d'une spécification internationale, alors même que les situations sont très contrastées. Ceci engendre des biais conséquents du fait de l'hétérogénéité des données en général ${ }^{(5)}$, et des disparités comptables, en particulier.

Ainsi, l'actuelle libéralisation des marchés conduit désormais les économistes à recentrer leur analyse sur les marchés qui émergent au sein des anciens monopoles nationaux intégrés. Il semble notamment particulièrement intéressant de tenir compte, dans l'étude des rendements d'échelle, de la séparation verticale mise en œuvre en Europe entre les activités de gestion de l'infrastructure et celles des services de transport. Si l'infrastructure est toujours considérée comme un unique monopole naturel ${ }^{(6)}$, la justification des monopoles dans les services de transport est étudiée avec d'autant plus d'acuité que ces activités s'ouvrent actuellement à la concurrence. Notre analyse s'inscrit donc dans un 
cadre nouveau, cohérent avec la réglementation européenne.

La justification d'éventuels monopoles naturels dans l'activité de transport requiert d'analyser les rendements d'échelle à un niveau suffisamment fin. En effet, s'il est imaginable que l'optimisation des rotations (du matériel et des personnels roulants) et la mutualisation de certaines tâches (achats, maintenance, administration) conduisent à certaines économies d'échelle, l'ampleur de celles-ci reste à estimer. A priori, l'optimum de production économique n'a aucune raison de coïncider avec les divisions administratives des régions, chargées de réguler les services ferroviaires dont elles ont la charge. Nous procédons donc à une étude microéconomique actualisée des rendements d'échelle, afin de remettre en cause certains préjugés sur ce sujet. Il ne s'agit donc pas d'une évaluation supplémentaire des économies d'échelle entre les grands réseaux européens, laquelle n'aurait plus beaucoup de sens. Ce travail prétend au contraire produire des résultats, dans un cadre actualisé, susceptibles d'orienter les choix des décideurs dans l'actuel contexte de libéralisation des marchés ferroviaires.

\section{Précisions théoriques}

Les rendements d'échelle sont classiquement définis comme étant l'inverse de l'élasticité apparente du coût à la production, estimée à partir d'une fonction de coût. Les rendements d'échelle peuvent toutefois également être calculés à partir des élasticités de la production aux facteurs, estimées par une fonction de production. Toutefois, il est plus pertinent de travailler sur une fonction de coût, dès que le volume de production est exogène, ce qui est bien souvent le cas dans les industries de réseau. Dans le transport ferroviaire de voyageurs, la production est évaluée par le trafic des personnes transportées ; on la mesure par le nombre de voyageurs.kilomètres.

\section{Les différentes formes de rendements d'échelle}

Dans les industries de réseau, les développements théoriques ont conduit à distinguer les rendements d'échelle selon qu'ils sont dus à une variation de trafic à réseau inchangé, ou à une variation de trafic induite par une modification du réseau. Ainsi, Caves et alii (1984), suivis par Pels et Rietveld (2000) et Seabright (2003), considèrent des fonctions de coût total du type $C\left(y_{i}, n, \ldots\right)$ dont les principales variables exogènes sont :

- les $y_{i}$ : les quantités de produit $i$

$-n$ : une variable traduisant l'étendue du réseau de transport.

Ils définissent alors :
- Les rendements de taille (RTS) : ils correspondent au sens courant des rendements d'échelle :

$R T S=\frac{1}{\sum_{i} \varepsilon_{y_{i}}+\varepsilon_{n}}$

où les $\varepsilon_{y_{i}}$ et $\varepsilon_{n}$ correspondent respectivement aux élasticités du coût aux produits et à celle du coût à la taille du réseau. Les rendements de taille sont croissants (ce qui correspond à la présence d'économies d'échelle) lorsque leur valeur est supérieure à 1 . Ils sont constants en cas d'égalité et décroissants, sinon. Lorsque ces rendements sont croissants sur un réseau caractérisé par des trafics $y_{i}$ et une étendue $n$, il n'y a aucun gain à partitionner ce réseau, toutes choses égales par ailleurs.

- Les rendements de densité (RTD) : ils ne font intervenir que l'élasticité du coût au trafic, toutes choses égales par ailleurs, c'est-à dire notamment à réseau fixé :

$R T D=\frac{1}{\sum_{i} \varepsilon_{y_{i}}}>R T S$

Pour cette raison, les rendements de densité ne peuvent être valablement estimés qu'à partir de données de panel, c'est-à-dire de séries de données chronologiques pour plusieurs réseaux. Les rendements de densité sont croissants (ce qui correspond à la présence d'économies d'échelle sur le seul trafic) lorsque leur valeur est supérieure à 1 . Ils sont constants en cas d'égalité et décroissants, sinon. Lorsque ces rendements sont croissants, il y a un gain à massifier les trafics sur ce réseau.

-Leséconomies d'envergure: elles sont susceptibles d'apparaître dans les entreprises multiproduits. Il s'agit du gain qui peut résulter de synergies entre les différentes activités (transport de marchandises et de voyageurs ou bien transport de voyageurs à grande vitesse et régional) :

$\operatorname{Scope}(i, j)=\frac{\partial^{2} C\left(y_{i}, y_{j}\right)}{\partial y_{i}, \partial y_{j}}$

Il y a des économies d'envergure entre les deux produits $i$ et $j$, lorsque ce terme (égal au coefficient du terme croisé d'une spécification translog) est négatif.

\section{Les fonctions de coût}

Les fonctions de coût définies dans la littérature (Braeutigam, 1999) résultent de la minimisation du coût sous contrainte de production. Elles intègrent donc en variables exogènes un vecteur des produits $y$ de l'entreprise et un vecteur des prix unitaires des facteurs de production $w$. Dans notre cas, la quasi-identité des prix unitaires des facteurs de 
production au sein de la $\mathrm{SNCF}^{(7)}$ permet de nous affranchir du vecteur de variables explicatives $w$, de toute façon indisponible. Ceci est d'autant plus justifié que notre analyse porte ici exclusivement sur l'évolution de la fonction de coût par rapport aux variables de production. Nous considérons donc les prix des facteurs comme étant fixés à des niveaux prédéterminés, ainsi que le suggère Varian (1992).

Précisons aussi que l'étude des rendements d'échelle nécessite de spécifier une fonction de coût de long terme. Une telle fonction correspond à la faculté qu'ont les entreprises d'ajuster tous leurs facteurs de production. Cette hypothèse est difficilement vérifiable sur un marché ferroviaire. Il y a en effet une suspicion permanente quant à l'adéquation des charges d'infrastructure, avec le maintien durable des performances de celle-ci. Lorsque les charges d'infrastructure reflètent mal les coûts de congestion, il faut interpréter prudemment les rendements de densité.

Suivant les recommandations de Caves et alii (1984), nous adoptons une fonction de coût qui intègre la dimension du réseau, afin de distinguer économies de taille et économies de densité. Nous initialisons nos estimations avec une forme fonctionnelle flexible, de type translog:

(1) $\ln C=\alpha_{0}+\beta_{V K} \ln V K+\beta_{L L} \ln L L+\gamma_{V K}(\ln V K)^{2}$

$$
+\gamma_{L L}(\ln L L)^{2}+\delta \ln V K \ln L L
$$

où $C$ correspond au coût total,

$V K$ désigne le trafic exprimé en voyageurs kilomètres,

$L L$ est la longueur de lignes du réseau exploité.

Le traitement de nos données fait appel à l'économétrie des données de panel, qui permet de prendre en compte la double dimension spatiotemporelle des observations. Nous rappelons brièvement dans les paragraphes qui suivent, les caractéristiques des estimateurs à notre disposition.

\section{Quelques rappels de méthodologie économétrique}

L'économétrie des données de panel, développée depuis les années soixante-dix, propose de nombreux modèles adaptés aux différents échantillons à analyser. L'objectif de ces modèles consiste à inclure dans les estimateurs, des effets propres aux différents individus ${ }^{(8)}$. Pour une présentation approfondie des éléments théoriques de l'économétrie des données de panel, voir Sevestre (2002).

Dans notre cas d'analyse, la nature des données (le fait que la variable longueur de lignes, $L L$, soit constante dans le temps) contraint le choix des modèles et estimateurs à notre disposition. En effet, ni les modèles à effets fixes, ni l'estimateur intra-individuel des modèles à erreur composée ne peuvent être mis en œuvre dans ce cas ${ }^{(9)}$. En revanche, les Moindres Carrés Quasi-Généralisés (MCQG) permettent d'estimer un modèle à erreur composée avec des variables exogènes constantes.

L'inconvénient majeur de cet estimateur est qu'il ne converge qu'à condition que les régresseurs soient exogènes : les effets spécifiques aléatoires doivent être indépendants des variables exogènes du modèle $^{(10)}$.

\section{Résultats économétriques}

Afin de traiter notre problématique de l'allotissement de façon exhaustive et pertinente, deux échantillons de données sont mobilisés. La section commence par une description des données avant d'exposer les résultats de l'estimation des fonctions de coût pour chacun de ces échantillons.

\section{Les données}

Les données que nous mobilisons proviennent de la comptabilité de la SNCF pour les services de transport régionaux de voyageurs ${ }^{(11)}$. À la source, nous disposons des coûts totaux et du trafic, par section de lignes régionales et par région administrative, sur la période 1992-1998. Toutefois, les données par section de ligne ne reflètent pas la logique de production des services de transport ferroviaire et n'ont, en ce sens, pas d'intérêt à l'état brut. Ainsi, il n'y a aucune pertinence à considérer séparément les coûts sur Strasbourg-Colmar, de ceux sur Colmar-Mulhouse, dans la mesure où une importante part du trafic est opérée par des trains Strasbourg-Colmar-Mulhouse. Une agrégation des données brutes a donc été menée afin de regrouper en « sous-réseaux » les différentes sections de ligne. Ces sous-réseaux constituent la plus petite unité qui puisse être pertinemment allotie compte tenu du trafic (contraintes de production et coûts d'interconnexion). Selon les cas, il s'agit d'une ligne ou d'un groupe de lignes d'une sous-région géographique, de sorte que les principaux flux de trafic actuels puissent toujours être acheminés sans correspondance.

À l'issue de ce travail, nous disposons donc des deux échantillons de données de panel suivants :

- un échantillon des 20 régions administratives qui correspond à 120 observations sur la période 1992-1998 (l'année 1996 est manquante). Cet échantillon permet d'évaluer les économies d'échelle sur les réseaux régionaux, et donc la pertinence d'un allotissement de ces réseaux ;

- un échantillon de 33 sous-réseaux répartis en Alsace, Bourgogne, Franche-Comté et Rhône-Alpes, qui conduit à 169 observations sur 1992-1998 (l'année 1996 est manquante et le panel 
Tableau 2 : statistiques descriptives des réseaux régionaux (120 observations)

\begin{tabular}{|l|c|c|c|c|}
\hline \multicolumn{1}{|c|}{ Variable } & Moyenne & Écart type & Minimum & Maximum \\
\hline Coûts (k euros 2004) & 78608 & 46091 & 21232 & 260946 \\
Voyageurs.kilomètres (millions) & 348 & 246 & 61 & 1245 \\
Longueur de lignes (km) & 1070 & 326 & 598 & 2033 \\
\hline
\end{tabular}

Source : SNCF.

Tableau 3 : statistiques descriptives des sous-réseaux (169 observations)

\begin{tabular}{|l|c|c|c|c|}
\hline \multicolumn{1}{|c|}{ Variable } & Moyenne & Écart type & Minimum & Maximum \\
\hline Coûts (k euros 2004) & 13,3 & 14,5 & 0,6 & 86,8 \\
Voyageurs.kilomètres (millions) & 55 & 73 & 1,4 & 393 \\
Longueur de lignes $(\mathrm{km})$ & 152 & 113 & 8 & 648 \\
\hline
\end{tabular}

Source : SNCF, RFF.

est non-cylindré). Cet échantillon vise à cerner les modalités d'un éventuel allotissement des réseaux régionaux.

Les statistiques descriptives de ces deux échantillons sont présentées respectivement dans les tableaux 1 et 2 .

\section{Estimations des fonctions de coût}

Nous présentons dans les paragraphes qui suivent la méthodologie économétrique appliquée à l'échantillon des réseaux régionaux, puis à celui des sous-réseaux; le détail des tests figure en annexe.

\section{Échantillon des réseaux régionaux}

L'estimation de la fonction translog complète (1) fournit des coefficients non significativement différents de zéro. Nous estimons donc une fonction réduite dans laquelle les coefficients $\beta_{V K}, \beta_{L L}$ et $\delta$ sont contraints, égaux à zéro. La spécification retenue s'écrit donc :

(2) $\ln C=\alpha_{0}+\gamma_{V K}(\ln V K)^{2}+\gamma_{L L}(\ln L L)^{2}$

Il s'agit de la spécification qui minimise le critère d'information de Akaike et maximise le $R^{2}$, parmi celles dont les coefficients sont significatifs. Un test du ratio de vraisemblance appliqué à cette restriction de trois degrés de liberté, la justifie pleinement : la spécification de la fonction de coût (2) doit être retenue.

Le test du multiplicateur de Lagrange confirme la présence d'effets spécifiques, et donc l'opportunité de traiter les données de panel en tant que telles. En revanche, le test d'Hausman ne conclut pas à la convergence de l'estimateur des MCQG : la variable $(\ln V K)^{2}$ est endogène aux effets spécifiques. Compte tenu de ce résultat et de la nature de nos données, les conditions nécessaires pour recourir à la méthode des variables instrumentales ne sont pas réunies. De ce fait, la dernière solution s'offrant à nous consiste à procéder en deux étapes, conformément à Hausman et Taylor (1981) :

- $\gamma_{V K}$ est d'abord estimé avec l'estimateur intra-individuel ;

$-\gamma_{L L}$ et $\alpha_{0}$ sont ensuite estimés avec l'estimateur inter-individuel (MCO appliqués aux moyennes individuelles) :

$\ln C_{i .}-\hat{\gamma}_{V K}\left(\ln V K_{i .}\right)^{2}=\alpha_{0}+\gamma_{L L}\left(\ln L L_{i}\right)^{2}+\varepsilon_{i .}$

Ce procédé présuppose l'exogénéité de la variable $(\ln L L)^{2}$ par rapport aux effets individuels, ce que nous ne pouvons tester. Toutefois, cette hypothèse ne semble pas trop forte dans la mesure où ces effets individuels associés aux coûts ont bien plus de raisons d'être liés au trafic qu'à la longueur du réseau.

Le tableau 4 ci-dessous présente les résultats de l'estimation en deux étapes de la fonction de coût (2). Les coefficients obtenus ne devraient pas être affectés par la multicolinéarité entre les deux variables exogènes ${ }^{(12)}$; ils sont significativement différents de 0 et du signe attendu.

Tableau 4 : estimation de la fonction de coût régionale (2) avec un modèle à erreur composée en deux étapes : estimateur intra-individuel $\left(\gamma_{V K}\right)$ puis inter-individuel $\left(\alpha_{0}, \gamma_{L L}\right)$.

\begin{tabular}{|ccc|}
\hline \multicolumn{3}{|c|}{$\begin{array}{c}\text { Observations : } 120 \\
\text { Variable endogène }: \ln \mathrm{C}\end{array}$} \\
\hline Paramètre & Coefficient & t-test \\
\hline$\alpha_{n}$ & 13,51 & 22,22 \\
$\gamma_{V K}$ & 0,01525 & 9,110 \\
$\gamma_{L L}$ & 0,04317 & 3,429 \\
\hline
\end{tabular}


À partir de la fonction translog complète (1), nous estimons une fonction réduite, dans laquelle le coefficient $\delta$ est contraint, égal à zéro. La spécification retenue s'écrit donc :

(3) $\ln C=\alpha_{0}+\beta_{V K} \ln V K+\beta_{L L} \ln L L+\gamma_{V K}(\ln V K)^{2}$

$$
+\gamma_{L L}(\ln L L)^{2}
$$

Il s'agit de la spécification qui minimise le critère d'information de Akaike et maximise le $R^{2}$, par rapport à la spécification complète. Un test du ratio de vraisemblance appliqué à cette restriction d'un degré de liberté, la justifie pleinement : le modèle contraint doit être retenu.

À l'échelle des sous-réseaux, les tests du multiplicateur de Lagrange nous conduisent à rejeter l'hypothèse de présence d'effets spécifiques en dimension temporelle, mais à conclure à l'existence d'effets spécifiques individuels. De plus, le test d'Hausman pour vérifier l'exogénéité des régresseurs est, cette fois, concluant.

Les résultats de cette estimation de la fonction de coût (3) figurent dans le tableau 5 ci-dessous.

Tableau 5 : estimation de la fonction de coût (3) des sous-réseaux par les MCQG

\begin{tabular}{|ccc|}
\hline \multicolumn{3}{|c|}{$\begin{array}{c}\text { Observations : } 169 \\
\text { Variable endogène }: \ln C\end{array}$} \\
\hline Paramètre & Coefficient & $t$-test \\
\hline$\alpha_{n}$ & 18,27 & 7,086 \\
$\beta_{V K}$ & $-1,074$ & $-3,465$ \\
$\beta_{I I}$ & 1,092 & 3,902 \\
$\gamma_{V K}$ & 0,04312 & 4,790 \\
$\gamma_{I I}$ & $-0,07802$ & $-2,646$ \\
$R^{2}$ & 0,941 & \\
\hline
\end{tabular}

Tous les coefficients sont significatifs à $5 \%$. Le signe négatif de $\beta_{V K}$ n'a rien d'incohérent, dans la mesure où, sur l'intervalle de fluctuation de nos variables, l'élasticité estimée coût-trafic demeure positive. Les variables exogènes introduites expliquent la quasi-totalité de la variance du coût $(94 \%)$; ici encore, le risque de multicolinéarité est faible.

\section{Interprétation économique}

Les fonctions de coût estimées à la section précédente permettent de calculer pour chaque échantillon les rendements d'échelle et les rendements de densité. Dans cette section, nous analysons dans un premier temps les économies d'échelle sur les réseaux régionaux, ce qui nous conduit à proposer l'allotissement de ces réseaux. Dans un second temps, nous dégageons quelques recommandations concernant la taille des sous-réseaux issus de l'allotissement.

\section{Les réseaux régionaux doivent-ils être allotis ?}

Les résultats présentés dans le tableau 4 de la section précédente permettent de calculer pour chacune de nos observations :

- les rendements de taille :

$$
R T S=\frac{1}{2(0,01525 \ln V K+0,04317 \ln L L)}
$$

- les rendements de densité :

$$
R T D=\frac{1}{2 \times 0,01525 \ln V K}
$$

Ces résultats conduisent à des rendements de densité estimés croissants et concaves, et ce, sans ambigüité : ils varient avec le trafic de 2,973 à 2,336, ce qui correspond à une moyenne de 2,620. En raisonnant en termes d'élasticité, cela signifie qu'une augmentation de $1 \%$ du trafic n'entraine qu'une augmentation de $0,38 \%$ des coûts. Ce résultat paraît tout à fait cohérent : une hausse du trafic n'engendre qu' une hausse des charges liées au matériel roulant ${ }^{(13)}$.

Ainsi, les rendements de densité calculés indiquent qu'il faut chercher à massifier au maximum le trafic tant qu'il n'y a pas congestion.

Les rendements de taille sont plus faibles, plutôt croissants et concaves : ils varient de 1,143 (Haute-Normandie 1994) à 0,960 (Rhône-Alpes 1998) pour une moyenne de 1,021 sur notre échantillon. Il apparaît toutefois que ces résultats ne sont pas significativement différents de 1 . Dès le seuil de confiance de $50 \%$, toutes les observations présentent des rendements d'échelle décroissants.

Du seul point de vue des économies d'échelle, l'absence de rendements de taille significativement croissants décrédibilise les arguments économiques en faveur d'un monopole d'envergure régionale. En intégrant le point de vue - plus large - de Gasmi et alii (2002), il est quasi certain que l'optimum collectif consiste dans un premier temps à allotir ces marchés régionaux. 
En effet, compte tenu des difficultés qu'ont les nouveaux entrants à pénétrer sur les marchés ferroviaires, il paraît nécessaire d'allotir les réseaux régionaux pour les rendre contestables et bénéficier ainsi des incitations concurrentielles. L'arrivée de concurrents privés devrait largement contribuer à améliorer l'efficience et réduire l'avantage informationnel de l'opérateur historique.

Dans Lévêque (2005), nous avons montré à l'aide d'une frontière stochastique que la rente informationnelle moyenne des TER de la SNCF représentait un surcoût supérieur à $10 \%$ par rapport au maximum d'efficience dont la SNCF est capable. Par ailleurs, les nouveaux entrants sur le marché du fret ferroviaire ont des coûts inférieurs d'un tiers à ceux de l'opérateur historique. L'économie potentielle offerte par la concurrence paraît ainsi très supérieure aux déséconomies d'échelle non significatives qui résulteraient d'un allotissement des réseaux régionaux.

L'analyse des deux facteurs principaux (économies d'échelle vs rentes informationnelles) illustre clairement le bénéfice collectif attendu de l'allotissement des réseaux régionaux. Ces réseaux ne sont donc pas des monopoles naturels, au sens précisé par Gasmi et alii (2002), ce qui suggère fortement de les allotir.

Afin d'orienter cet allotissement, il reste à discuter la taille pertinente des sous-réseaux en intégrant les deux autres facteurs de décision : les coûts d'interconnexion et les coûts de transaction.

\section{Quelle taille pertinente pour un sous-réseau ferroviaire?}

Les sous-réseaux constitués à la partie précédente sont les plus petits qui puissent l'être en intégrant les coûts d'interconnexion, c'est-à-dire en évitant que les principaux flux de trafic fassent l'objet d'une correspondance. À l'exception de voyageurs aux parcours tortueux, cette proposition d'allotissement

Figure 1 : nomogramme des rendements de taille pour les sous-réseaux : trafic en fonction de la longueur de lignes

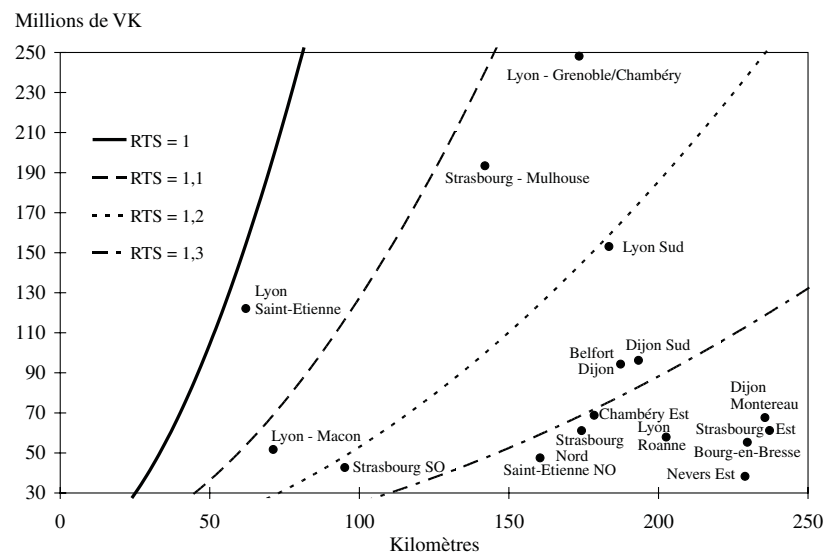

ne devrait donc pas réduire sensiblement les externalités de réseau.

En revanche, le nombre de sous-réseaux constitués pourrait entraîner des coûts de transaction un peu trop élevés à court terme. Avec 9 sous-réseaux en Alsace, 8 en Bourgogne, 4 en Franche-Comté et 12 en Rhône-Alpes, cette proposition ne semble cohérente avec les moyens dont dispose chacune de ces régions qu'à plus long terme. Rappelons toutefois que parmi ces sous-réseaux, les isolats (constitués d'une ou plusieurs petites lignes isolées du réseau principal) peuvent être régulés moyennant des coûts de transaction très faibles.

Dans les paragraphes qui suivent, nous analysons les rendements d'échelle sur ces sous-réseaux. Cela conduit à déterminer si le regroupement de certains d'entre eux n'apporterait pas quelques économies d'échelle en plus d'une réduction des coûts de transaction.

Les résultats présentés dans le tableau 4 de la section précédente permettent de calculer pour chacune de nos observations :

- les rendements de taille :

$$
R T S=\frac{1}{-1,074+1,092+2(0,04312 \ln V K-0,07802 \ln L L)}
$$

- les rendements de densité :

$$
R T D=\frac{1}{-1,074+2 \times 0,04312 \ln V K}
$$

Ces résultats conduisent à des rendements de densité estimés toujours largement supérieurs à 1 (2,7 en moyenne), ce qui incite fortement à massifier les trafics sur les différents sous-réseaux tant qu'il n'y a pas de congestion. Ces valeurs confirment l'importance des économies de densité à l'échelle d'une ou de quelques lignes.

Figure 2 : nomogramme des rendements de taille pour les sous-réseaux. Détail des plus petits réseaux : trafic en fonction de la longueur de lignes

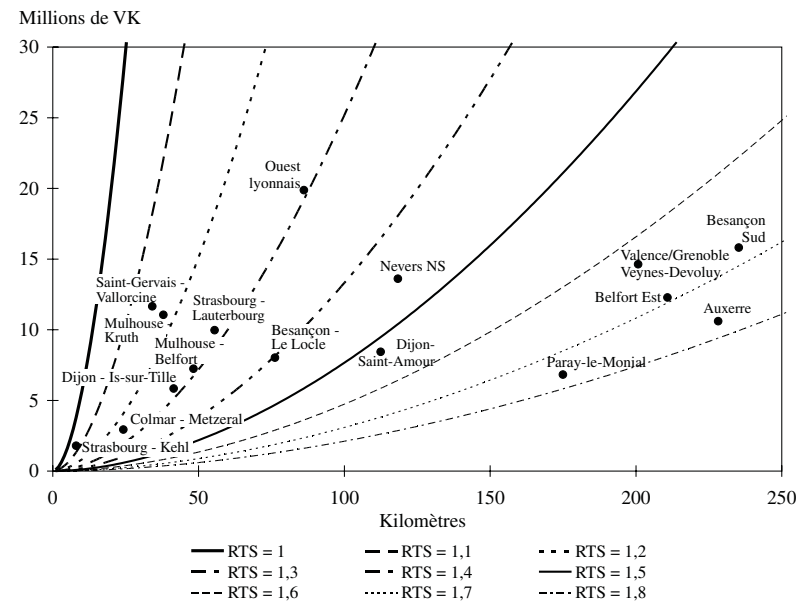


Les rendements de taille varient, eux, de 1,77 (réseau de Paray-le-Monial ; 1995) à 1,02 (ligne Lyon Saint-Étienne ; 1993). Ils sont significativement croissants sur les sous-réseaux susceptibles d'être encore partitionnés ; il n'apparaît donc pas intéressant de segmenter davantage les réseaux régionaux. Au contraire, il peut être pertinent de regrouper certains de ces sous-réseaux, afin de bénéficier davantage des économies d'échelle. Cela permettrait simultanément de réduire les coûts de transaction et d'accroître à la marge les effets de réseau, tout en assurant la contestabilité des marchés.

Les nomogrammes suivants (figures 1 et 2) permettent de repérer sur une échelle large et sur une autre plus détaillée, les rendements de taille pour les différents sous-réseaux. Chacun des sous-réseaux est caractérisé par sa longueur (en abscisse) et son trafic en 1998 (en ordonnée); les courbes de niveaux indiquent la valeur des rendements de taille. On retrouve sur la première figure la ligne Lyon Saint-Étienne dont les rendements de taille sont quasi constants (proches de 1) : un accroissement de la longueur du réseau et du trafic se répercutera proportionnellement sur les coûts, c'est-à-dire que les rendements d'échelle y sont déjà totalement consommés. À l'opposé, sur la seconde figure, le réseau de Paray-le-Monial présente de forts rendements de taille : une hausse de la longueur du réseau et surtout du trafic augmenterait les coûts relativement faiblement, les rendements d'échelle jouant à plein.

\section{Conclusion : proposition d'aide à la décision}

Avant de conclure sur les principaux résultats obtenus et d'en tirer quelques recommandations, il convient de ne pas perdre de vue que ceux-là demeurent endogènes à la position monopolistique de la SNCF. L'apparition sur les marchés de nouveaux entrants avec des techniques de production différentes va modifier les fonctions de coût estimées et donc les rendements d'échelle calculés.

Ces résultats sont également tributaires de la qualité des données comptables sur lesquelles ils s'appuient, de même que toute analyse de rendements d'échelle. En l'occurrence, si l'affectation des différentes charges par le logiciel comptable FC12K paraît acceptable pour les réseaux régionaux, elle paraît plus discutable pour les sections de ligne. En effet, il est permis de douter de l'attribution de certaines charges communes (accompagnement, manœuvre, structure) à telle ou telle portion de ligne. Il semble néanmoins que les imperfections associées à la répartition des charges communes par le $\mathrm{FC} 12 \mathrm{~K}$ sont moins problématiques que les biais introduits dans les estimations internationales par l'hétérogénéité des données.

Les résultats obtenus dans la première section de la précédente partie plaident en faveur d'un certain allotissement des actuels réseaux régionaux. Cela permettrait de favoriser l'arrivée de nouveaux entrants et donc de bénéficier d'une réduction des rentes informationnelles, sans engendrer pour autant de déséconomies d'échelle significatives. La proposition d'allotissement dans la deuxième section de la précédente partie permet de discerner les facteurs clés de la taille pertinente des sous-réseaux. Si les coûts d'interconnexion induits paraissent marginaux, il convient de veiller à ce que les coûts de transaction restent limités. Compte tenu des rendements d'échelle, il semble cohérent de proposer aux régions d'allotir leur réseau en 3 ou 10 sous-réseaux, en fonction de leur taille.

Cette proposition d'allotissement incite les pouvoirs publics français à libéraliser au plus tôt les services régionaux de transport ferroviaire, anticipant avec bon sens un prochain règlement européen. Alors que les régions déplorent leur capture par l'opérateur historique et les coûts des TER, l'allotissement leur permettrait de mieux réguler ces services, à l'image des Länder allemands. Depuis 1993, les Länder ont la possibilité d'ouvrir à la concurrence leur réseau régional. Ceux qui ont libéralisé leur réseau ont lancé des appels d'offre sur des sous-réseaux d'une longueur de $200 \mathrm{~km}$ en moyenne. Cela a permis à la concurrence de s'implanter progressivement (plus de $50 \%$ des appels d'offre remportés depuis 1996), sans générer de perte de surplus particulière (déséconomies d'échelle, coûts d'interconnexion, coûts de transaction).

Ainsi en France, il conviendrait dans un premier temps de déléguer quelques sous-réseaux, notamment les isolats, que les nouveaux entrants seraient susceptibles de remporter. Cela permettra de limiter les coûts d'interconnexion et les coûts de transaction, tout en bénéficiant de la réduction des rentes informationnelles, induite par la concurrence. Avec l'arrivée progressive des nouveaux entrants sur les marchés, les sous-réseaux allotis pourraient être élargis, tout en demeurant contestables.

Mais comme en Allemagne, cette évolution devrait être très progressive ; d'ici là, le pouvoir de marché de l'opérateur historique demeurera important. Afin de le contrôler, les régulateurs régionaux peuvent d'ores et déjà mettre en œuvre un mécanisme incitatif de concurrence par comparaison tel celui proposé dans Lévêque (2005). 


\section{Notes}

(1) Hors Corse et Île-de-France dont les services de transport régional sont très différents.

(2) Les auteurs justifient notamment ce résultat par la possibilité de mettre en œuvre la concurrence par comparaison sur les marchés duopolistiques.

(3) Dans un premier temps, compte tenu des multiples barrières à l'entrée (qu'elles soient d'ordre technique, social ou réglementaire) et de l'avantage informationnel considérable de l'opérateur historique, les nouveaux entrants ne peuvent prétendre gérer dans de bonnes conditions l'ensemble d'un réseau régional. Voir à ce sujet Gauthier-Lescop et Lévêque (2006).

(4) Encore faut-il prouver l'existence même de rendements d'échelle croissants dans ces activités de service de transport.

(5) Dans plusieurs pays, certains trafics - souvent les moins rentables pour l'opérateur historique - ont été captés par des concurrents dont les fonctions de coût sont très différentes.

(6) Malgré l'émergence croissante des gestionnaires d'infrastructure dans l'industrie ferroviaire européenne, les données manquent pour évaluer finement les rendements d'échelle dans ces activités. Actuellement, seuls des arguments en termes d'indivisibilités d'offre justifient l'actuelle organisation monopolistique de ces activités. Il a cependant été prouvé dans l'industrie des télécommunications (en théorie - voir Gasmi et alii (2002) -, comme en pratique) que les raisonnements fondés sur les indivisibilités conduisaient à des conclusions erronées. . .

(7) Ceci est garanti par la gestion centralisée de la SNCF. Ainsi, à grade et échelon égaux, le coût du travail est le même pour tous les cheminots, à l'exception de la majoration résidentielle, susceptible d'augmenter de $2 \%$ à $4 \%$ le traitement suivant la commune d'affectation du poste.

(8) Nous ne présentons ici que les modèles à effets individuels et non temporels, dans la mesure où la présence d'effets temporels dans nos observations n'est pas avérée.

(9) Les modèles à effets fixes caractérisent les individus par une constante propre à chacun; de ce fait, lorsqu'une variable exogène est constante dans le temps, elle est une combinaison linéaire des effets fixes individuels et le modèle n'est plus identifiable. Par ailleurs, l'estimateur intra-individuel qui repose sur le calcul de la différence entre les observations et leur moyenne individuelle ne s'applique pas aux variables constantes dans le temps dont la variabilité intra-individuelle est nulle pour chaque individu.

(10) Dans ce cas, la distribution asymptotique de cet estimateur est la même que celle de l'estimateur intra-individuel. Aussi, afin de tester la convergence de l'estimateur des MCQG, Hausman et Taylor (1981) ont proposé un test, dit " de Hausman ", qui compare cet estimateur et sa matrice des variances-covariances aux caractéristiques de l'estimateur intra-individuel. Lorsqu'une variable est constante dans le temps, le test d'Hausman porte sur les estimations des coefficients associés aux autres variables.

(11) Il s'agit des résultats fournis par le logiciel comptable FC12K. Les variables de coûts que nous mobilisons résultent donc en partie de l'allocation comptable d'un certain nombre de charges communes. De ce fait, ces « coûts » (qui n'en sont pas réellement) sont affectés par les différences régionales d'effort de la SNCF, mais pas par les rentes informationnelles que la SNCF peut dégager lors de ses négociations avec les régions (Lévêque, 2005).

(12) Greene (1997) rappelle la règle de Klein suivant laquelle le coefficient de détermination de la régression entre les variables exogènes $\left(R_{V K / L L}^{2}=30 \%\right)$ doit être inférieur à celui de la régression principale $\left(R_{C / V K, L L}^{2}=94 \%\right)$ pour éviter de se soucier de la multicolinéarité.

(13) Lorsque le trafic augmente, il finit par devenir nécessaire de recourir à du matériel roulant plus lourd et plus coûteux ; on passe d'un automoteur avec un élément à un automoteur avec plusieurs éléments, puis à une rame tractée par une locomotive. Cependant, les charges de conduite demeurent inchangées, tandis que les charges d'énergie et d'infrastructure n'augmentent que marginalement (en l'absence de congestion).

(14) Le test de Honda, présenté par Sevestre (2002), qui est un peu plus restrictif que celui du multiplicateur de Lagrange est tout aussi concluant. 


\section{Bibliographie}

Braeutigam R.R. (1989). « Optimal Policies for Natural Monopolies ", in Handbook of Industrial Organization, North-Holland, pp. 1290-1347.

Braeutigam R.R. (1999). " Learning about Transport Costs », in Essays in transportation economics and policy: A handbook in honor of John R. Meyer, Brookings Institution, pp. 57-97.

Cantos Sánchez P. (2001). «Vertical Relationships for the European Railway Industry », Transport Policy, vol. 8, $\mathrm{n}^{\circ} 2$, pp. 77-83.

Caves D.W., Christensen L.R. et Tretheway M.W. (1984).

«Economies of Density Versus Economies of Scale : why Trunk and Local Service Airline Costs Differ », Rand Journal of Economics, vol. 15, n 4, pp. 471-489.

Caves D.W., Christensen L.R., Tretheway M.W. et Windle R.J. (1985). " Network Effects and the Measurement of Returns To Scale and Density for U.S. Railroads ", in Analytical Studies in Transport Economics, Cambridge University Press, pp. 97-120.

Gasmi F., Laffont J.-J. et Sharkey W.W. (2002). « The Natural Monopoly Test Reconsidered: an Engineering Process-Based Approach To Empirical Analysis in Telecommunications ", International Journal of Industrial Organization, vol. 20, n 4, pp. 435-459.

Gauthier-Lescop L. et Lévêque J. (2006). « De la régionalisation à la concurrence régulée. Analyse économique et juridique de la future organisation du transport ferroviaire régional de voyageurs », Politique et Management Public, vol. $24, \mathrm{n}^{\circ} 1$, pp. 1-28.

Greene W.H. (1997). Econometric Analysis, Prentice-Hall.

Hausman J.A. et Taylor W.E. (1981). " Panel Data and Unobservable Individual Effects ", Econometrica, vol. 49, $\mathrm{n}^{\circ}$ 6, pp. 1377-1398.

Ida T. et Suda M. (2004). " The Cost Structure of the Japanese Railway Industry : The Economies of Network Density and of Scope and the Cost Gap Between Japan's Regional Railways After Privatization », International Journal of Transport Economics, vol. 31, n 1, pp. 23-37.

Lévêque J. (2005). " Réduire le poids des contraintes informationnelle, politique et sociale grâce à la concurrence par comparaison : Le cas des trains régionaux de la SNCF », Revue d'économie Industrielle, $\mathrm{n}^{\circ} 111$, pp. 57-78.

Pels E. et Rietveld P. (2000). « Cost Fuctions in Tranport», in Handbook of Transport Modelling, Pergamon, pp. 321-333.

Seabright P. (2003). « The Economics of Passenger Rail Transport : A Survey », Working Paper, $n^{\circ} 163$, IDEI.

Sevestre P. (2002). Économétrie des données de panel, Dunod.

Varian H.R. (1992). Introduction à la microéconomie, De Boeck Université.

\section{Annexe : tests économétriques}

\section{Tests portant sur l'échantillon des réseaux régionaux}

Spécification de la fonction de coût : il s'agit de tester l'hypothèse nulle :

$H_{0}: \beta_{V K}=\beta_{L L}=\delta=0$

contre l'hypothèse :

$H_{1}: \beta_{V K}=\beta_{L L}=\delta \neq 0$

Le ratio de vraisemblance $L R=-2\left[\ln L\left(H_{0}\right)-\ln L\left(H_{1}\right)\right]$ suit une loi du $\chi^{2}$ à 3 degrés de liberté. Le calcul donne :

$L R=-2(77,40-78,27)=1,73<7,81=\chi_{3,5 \%}^{2}$

Ainsi, pour les valeurs usuelles du risque de rejet à tort (5\%), la spécification contrainte doit être retenue.

Présence d'effets spécifiques: le test du multiplicateur de Lagrange ( $L M$-test), proposé par Breush et Pagan évalue l'hypothèse nulle :

$H_{0}$ : absence d'effets spécifiques

contre l'hypothèse :

$H_{1}$ : présence d'effets spécifiques.

Sous $H_{0}$, la statistique du multiplicateur de Lagrange suit asymptotiquement une loi du $\chi^{2}$ à 1 degré de liberté :

$L M=\frac{N T}{2(T-1)}\left[\frac{\sum_{n=1}^{N}\left(\sum_{t=1}^{T} \hat{\varepsilon}_{n, t}\right)^{2}}{\sum_{n=1}^{N}\left(\sum_{t=1}^{T} \hat{\varepsilon}_{n, t}^{2}\right)}-1 \mid \rightarrow \chi_{1}^{2}\right.$

Ce test nous conduit à rejeter l'hypothèse de présence d'effets spécifiques en dimension temporelle : $L M=0,93<3,84=\chi_{1,5 \%}^{2}$. En revanche, le même test permet de conclure à l'existence d'effets spécifiques individuels $^{(14)}: L M=179,66 \gg>3,84=\chi_{15 \%}^{2}$.

Exogénéité des régresseurs : le test d'Hausman évalue l'hypothèse nulle :

$H_{0}: \gamma_{\text {within }}$ et $\gamma_{M C Q G}$ sont convergents,

où $\gamma_{\text {within }}$ correspond au vecteur des estimateurs intra-individuels,

et $\gamma_{M C Q G}$ correspond au vecteur des estimateurs des MCQG. contre l'hypothèse :

$H_{1}: \gamma_{\text {within }}$ est convergent et $\gamma_{M C O G}$ est non-convergent.

Le logiciel Limdep effectue ce test en comparant l'estimateur des MCQG à l'estimateur intra-individuel appliqué au modèle incluant une variable indépendante du temps : $(\ln L L)^{2}$. Pour respecter le cadre d'application spécifié par Hausman et Taylor, il faut programmer un test portant uniquement sur $(\ln V K)^{2}$. Il s'agit de calculer la statistique de Hausman, qui suit asymptotiquement une loi $\mathrm{du} \chi^{2}$ à $\operatorname{dim}(\gamma)=1$ degré de liberté :

$Q_{H}=\left(\hat{\gamma}_{\text {within }}-\hat{\gamma}_{M C Q G}\right)^{\prime}\left[\hat{V}\left(\hat{\gamma}_{\text {within }}\right)-\hat{V}\left(\hat{\gamma}_{M C Q G}\right)\right]^{-1}$

$\left(\hat{\gamma}_{\text {within }}-\hat{\gamma}_{M C Q G}\right) \rightarrow \chi_{1}^{2}$ 
où $\hat{V}($.$) désigne la matrice des variances-covariances$ estimées.

Le calcul donne : $Q_{H}=11,4>3,84=\chi_{1,5 \%}^{2}$; il faut donc rejeter l'hypothèse de convergence $H_{0}$.

\section{Tests portant sur l'échantillon des sous-réseaux}

Spécification de la fonction de coût : le calcul du ratio de vraisemblance donne :

$L R=-2(12,9501-13,0352)=0,1702<<3,84=\chi_{1,5 \%}^{2}$. Ainsi, pour les valeurs usuelles de rejet à tort, la spécification contrainte doit être retenue.

Présence d'effets spécifiques : le test du multiplicateur de Lagrange conduit à rejeter l'hypothèse de présence d'effets spécifiques en dimension temporelle : $L M=0,01<<3,84=\chi_{1,5 \%}^{2}$. En revanche, il permet de conclure à l'existence d'effets spécifiques individuels : $L M=259,40 \gg>3,84=\chi_{1,5 \%}^{2}$.

Exogénéité des régresseurs : la programmation du test d'Hausman porte uniquement sur $\ln V K$ et $(\ln V K)^{2}$; la statistique de Hausman suit asymptotiquement une loi du $\chi^{2}$ à $\operatorname{dim}(\gamma)=2$ degrés de liberté. Le calcul donne : $Q_{H}=2,20<5,99=\chi_{2,5 \%}^{2}$, ce qui permet de recourir à l'estimateur des $M C Q G$. 
